\title{
Kinematic and thermal structure at the onset of high-mass star formation - ISOSS23053
}

\author{
Simon Bihr ${ }^{1}$, Henrik Beuther ${ }^{1}$, Hendrik Linz ${ }^{1}$, Sarah E. Ragan ${ }^{2}$, \\ Jochen Tackenberg ${ }^{1}$, Rowan J. Smith ${ }^{3}$, Thomas Henning ${ }^{1}$ and Oliver \\ Krause $^{1}$ \\ ${ }^{1}$ Max Planck Institute for Astronomy, \\ Königstuhl 17, 69117 Heidelberg, Germany \\ email: name@mpia.de \\ ${ }^{2}$ School of Physics \& Astronomy, The University of Leeds, \\ E.C. Stoner Building, Leeds, LS2 9JT, UK \\ email: s.ragan@leeds.ac.uk \\ ${ }^{3}$ Jodrell Bank Centre for Astrophysics, University of Manchester, \\ Oxford Road, Manchester, M13 9PL, UK \\ email: rowanjsmith.astro@googlemail.com
}

\begin{abstract}
Within the 'earliest phases of star formation' (EPoS) Herschel project, we observed the $\mathrm{NH}_{3}$ inversion lines of 6 very young high-mass star-forming regions at high spatial resolution $\left(3-5^{\prime \prime}\right)$ with the JVLA. While the Herschel data provide details about the dust continuum, the ammonia data reveal the kinematics and temperatures. Here we present the exciting star-forming clump ISOSS23053 that shows multiple velocity components. We observe a prominent velocity step within the clump, which could be a sign of colliding or converging flows that triggers star formation. Furthermore, we used the JVLA in the C-array configuration to study this source in more detail and we present the first results from the new data. They support the idea of converging flows, as we observe two components in the $\mathrm{NH}_{3}(1,1)$ and $(2,2)$ line, whereas the higher excited $\mathrm{NH}_{3}(3,3)$ line shows one component that links the two lower excited lines.
\end{abstract}

Keywords. Stars: formation - Stars: massive - ISM: clouds - ISM: kinematics and dynamics

\section{Introduction}

Even though the understanding of high-mass star formation has made tremendous progress over the past decade (e.g., Zinnecker \& Yorke 2007; Tan et al. 2014), the initial conditions are still poorly constrained. The most prominent candidates for young massive clumps are the infrared dark clouds (IRDCs). With the Herschel bolometers, we encompass the transition from continuum absorption to emission in these objects, but such observations lack any kinematic information. As the formation process of stars is thought to be highly dynamic (e.g., Bonnell et al. 2004; Klessen et al. 2005), such information is crucial for constraining star formation theories. Follow-up observations of molecular lines are mandatory for investigating the kinematics of star-forming regions. For this project, we observed the $\mathrm{NH}_{3}(1,1)$ and $\mathrm{NH}_{3}(2,2)$ inversion transitions of ammonia because they probe the temperature and kinematics of dense gas (e.g., Ragan et al. $2012 b)$. We selected 6 very young high-mass star-forming clumps from the Herschel EPoS sample (Ragan et al. 2012a) to map with the JVLA in the D (most compact) configuration. Within the velocity maps, we find signatures which may be linked to converging flows. The results of this sample are given in Bihr et al. (2015). Due to the low spectral 

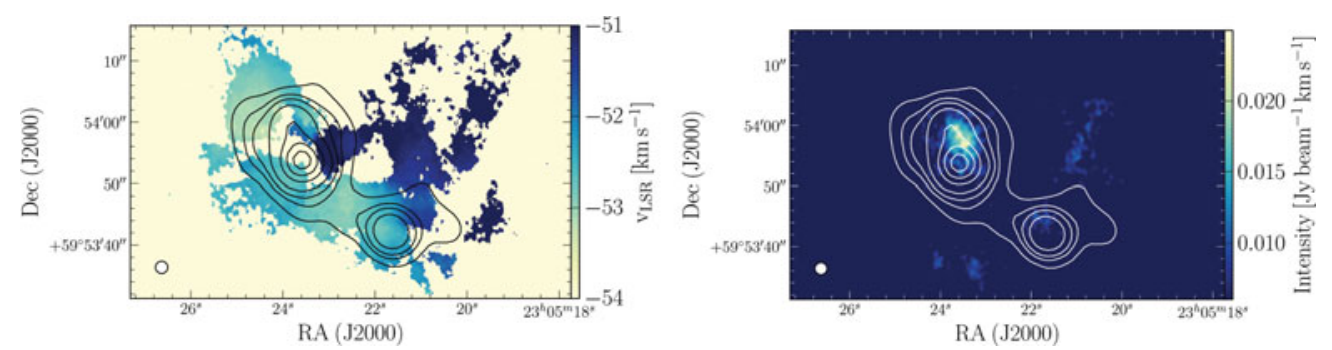

Figure 1. The left panel shows the velocity structure of the $\mathrm{NH}_{3}(1,1)$ line using the JVLA C-array data. The right panel presents the integrated intensity of the higher excited $\mathrm{NH}_{3}(3,3)$ line of the JVLA C-array data. In both panels, the contours show Herschel $70 \mu \mathrm{m}$ emission.

resolution of these early JVLA observations, we were not able to resolve the lines and we could not determine the line width accurately. Hence, we observed two sources of our sample again with the JVLA in C-array to achieve a higher spatial, but also spectral resolution. In this proceeding, we present this data for the clump ISOSS23053.

\section{ISOSS23053}

The clump ISOSS23053 has a mass of M 500-900M $\odot$ (Ragan et al. 2012a) and shows several indicators of star formation, such as high density dust peaks, spectral signatures of infall and signs of outflows (Birkmann et al. 2007). The infrared observations reveal two emission peaks at $70 \mu \mathrm{m}$ (see contours in Fig.1). In contrast to this, the ammonia observations reveal a more clumpy structure and they show a steep velocity step with two components, revealing a difference of $\sim 1.5 \mathrm{~km} \mathrm{~s}^{-1}$. As seen in the left panel of Fig. 1 , the velocity step is situated close to the far-IR emission peaks. The exciting question is, if this velocity step traces a converging flow, which triggers the observed star formation activity. Our previous observations (Bihr et al. 2015) were not able to answer this question unambiguously, as the spectral and spatial resolution was not sufficient. However, new observations at higher spectral resolution allow us to study this object in great detail. Here, we want to highlight the preliminary analysis of the higher excited $\mathrm{NH}_{3}(3,3)$ line, which is shown in the right panel of Fig.1. We observe a clear detection of this higher excited line at the location of the velocity step, which indicates higher kinetic temperature. In contrast to the two velocity components seen in the $\mathrm{NH}_{3}(1,1)$ and $\mathrm{NH}_{3}(2,2)$ lines, we detect a single component in the $\mathrm{NH}_{3}(3,3)$ line, which spans over both velocity components of the lower excited lines. This is another indicator that we see an interaction between the two velocity components, which may trigger the observed star formation activities.

\section{References}

Bihr, S., Beuther, H., Linz, H., et al. 2015, A\&A, 579, A51

Birkmann, S. M., Krause, O., Hennemann, M., et al. 2007, A\&A, 474, 883

Bonnell, I. A., Vine, S. G., \& Bate, M. R. 2004, MNRAS, 349, 735

Klessen, R. S., Ballesteros-Paredes, J., Vázquez-Semadeni, E., \& Durán-Rojas, C. 2005, ApJ, 620,786

Ragan, S., Henning, T., Krause, O., et al. 2012a, A\&A, 547, A49

Ragan, S. E., Heitsch, F., Bergin, E. A., \& Wilner, D. 2012b, ApJ, 746, 174

Tan, J. C., Beltrán, M. T., Caselli, P., et al. 2014, Protostars and Planets VI, 149

Zinnecker, H. \& Yorke, H. W. 2007, ARA\&A, 45, 481 